\title{
Quasi-optimization of the slender geometrically nonlinear supporting system with non-prismatic element under the specific load
}

\author{
Janusz Szmidla ${ }^{1}$, and Anna Jurczyńska, ${ }^{2, *}$ \\ ${ }^{1}$ Institute of Mechanics and Machine Design Fundamentals, Częstochowa University of Technology, \\ Częstochowa, Poland \\ ${ }^{2}$ Institute of Mechanics and Machine Design Fundamentals, Częstochowa University of Technology, \\ Częstochowa, Poland
}

\begin{abstract}
This paper is devoted to the issue of free vibrations of a geometrically nonlinear column with a nonprismatic element modelling the supporting structure subjected to the specific load. The boundary problem was formulated on the basis of the Hamilton's principle and its solution was obtained using the Lindstedt - Poincare's small parameter method (a perturbation method). Within the range of the kinetic criterion of the static equilibrium, an influence of the shape of a rod of variable crosssection on the values of free vibrations and the bifurcation load of the system was determined. Presented quasi-optimization issue has been reduced to defining the ranges of physical and geometrical parameters where an increase of the bifurcation load is the biggest in comparison with the reference system.
\end{abstract}

\section{Introduction}

Slender systems are commonly used as a components of many structures, among others in machinery construction, civil engineering or mining industry. Widespread application forces researchers to predict their behaviour and strength (stability). Studies on controlling their dynamic properties and the issue of optimization are of particular importance.

Work [1] concerns the influence of the size of the amplitude parameter on the natural frequency of the partially compressed slender column under the Euler's load. The problem of optimization of a similar system with regard to the occurrence of cracks is described in [2], where genetic algorithms were used. The topic of work [3] is the solution of the problem of a dynamic stability in stepped cantilever beam with attached discrete elements obtained by applying the mode summation method. The original optimization algorithm was proposed in the works $[4,5]$.

This paper proposes a quasi-optimization algorithm in relation to the control of natural vibration frequencies of the geometrically nonlinear slender system under the follower force directed towards the positive pole (a specific load). 


\section{Boundary problem formulation}

A geometrically nonlinear column $\mathbf{N}$ built of three prismatic rods connected at the free ends presented in figure $1 \mathrm{~b}$ is considered in the paper. The relation between the flexural stiffness of the particular rods is given by the coefficient of the flexural stiffness asymmetry $\mu$ while the total flexural stiffness is assumed to be constant:

$$
\mu=\frac{(E J)_{I I I}}{(E J)_{I}+(E J)_{I I}}, \quad \sum_{k=I}^{I I I}(E J)_{k}=\text { const. }
$$

In the target system NN (fig. 1a) the internal element was replaced by the nonprismatic rod of the same volume, resulting from the value of the coefficient $\mu$. The variable crosssection was modelled by the division of the rod into the finite number of prismatic segments of the same length and thick, which widths were described by the approximating functions (fig. 1c). The calculations were limited to the shape approximation by means of the polynomial of degree I and II.

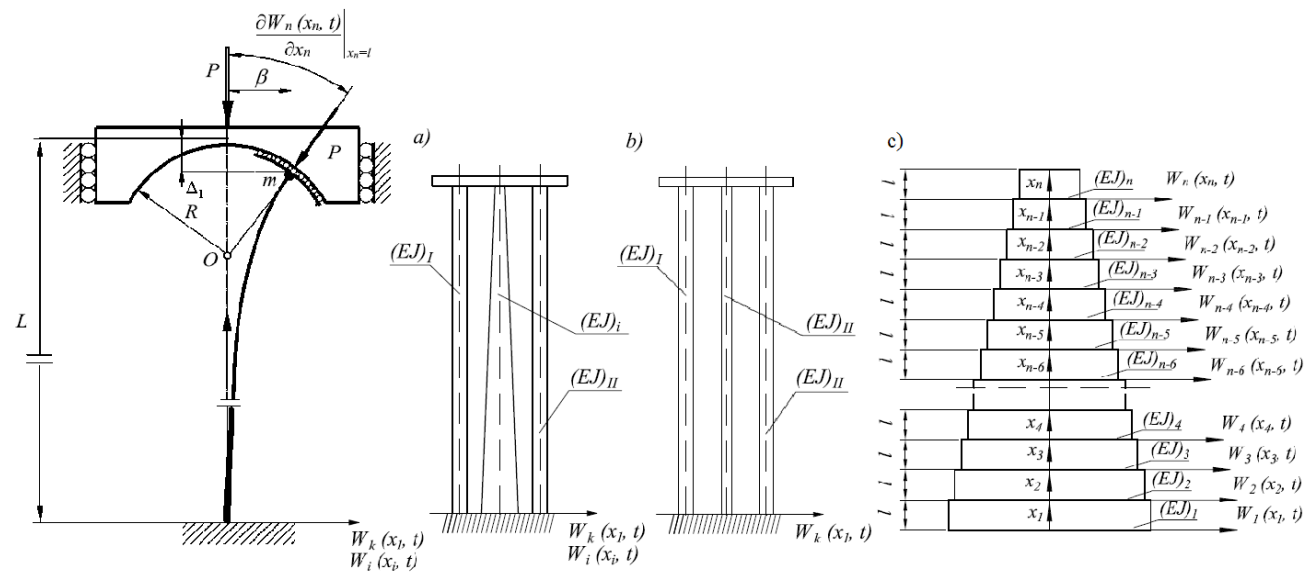

Fig. 1. The physical model of the geometrically nonlinear column a) column with the nonprismatic element, b) the comparative column, c) the model of the rod of variable cross-section.

The columns were subjected to the follower force directed towards the positive pole, realized through the loading structure built of elements of circular outlines (parts of the rolling bearing). The applied load is a case of the specific load defined by L. Tomski [6] and it combines features of the follower force (nonconservative) and the force directed towards the pole. The load is transmitted from the loading head to the receiving head and then via a rigid element (enabling correct mounting of the column) on the system. The advantage of using systems under the specific load as a supporting structure is obtaining higher values of the bifurcation and critical loads in comparison with the classical Euler's load.

Taking into account the Bernoulli-Euler's theory and described physical models, the total potential energy $V$ as well as the kinetic energy $T$ (comp. $[7,8]$ ) of the analyzed system were defined. The boundary problem was formulated on the basis of the Hamilton's principle (principle of stationary action) using the calculus of variations:

$$
\int_{t_{1}}^{t_{2}}(\delta T-\delta V) d t=0
$$

The differentia equations of motion in the transverse (eq. 4) and longitudinal (eq. 5-6) directions obtained based on the relation (3) may be written as follow: 


$$
\begin{gathered}
(E J)_{i} \frac{\partial^{4} W_{i}\left(x_{i}, t\right)}{\partial x_{i}^{4}}+S_{I I I}(t) \frac{\partial^{2} W_{i}\left(x_{i}, t\right)}{\partial x_{i}^{2}}+(\rho A)_{i} \frac{\partial^{2} W_{i}\left(x_{i}, t\right)}{\partial t^{2}}=0, \quad i=1,2, \ldots, n . \\
(E J)_{k} \frac{\partial^{4} W_{k}\left(x_{k}, t\right)}{\partial x_{k}^{4}}+S_{k}(t) \frac{\partial^{2} W_{k}\left(x_{k}, t\right)}{\partial x_{k}^{2}}+(\rho A)_{k} \frac{\partial^{2} W_{k}\left(x_{k}, t\right)}{\partial t^{2}}=0, \quad k=I, I I, \\
U_{i}\left(x_{i}, t\right)-U_{i}(0, t)=-\frac{S_{i}(t)}{(E A)_{i}} x_{i}-\frac{1}{2} \int_{0}^{x_{i}}\left(\frac{\partial W_{i}\left(x_{i}, t\right)}{\partial x_{i}}\right)^{2} d x_{i}, \quad i=1,2, \ldots, n, \\
U_{k}\left(x_{k}, t\right)=-\frac{S_{k}(t)}{(E A)_{k}} x_{k}-\frac{1}{2} \int_{0}^{x_{k}}\left(\frac{\partial W_{k}\left(x_{k}, t\right)}{\partial x_{k}}\right)^{2} d x_{k}, \quad k=I, I I .
\end{gathered}
$$

The geometrical and natural boundary conditions describing the column, including the continuity conditions relate to the particular segments of the nonprismatic rod, are given by the following formulas:

$$
\begin{aligned}
& W_{I}(0, t)=W_{I I}(0, t)=W_{1}(0, t)=0, \\
& W_{I}(L, t)=W_{I I}(L, t)=W_{n}(l, t), \\
& \left.\frac{\partial W_{I}\left(x_{I}, t\right)}{\partial x_{I}}\right|_{x_{I}=0}=\left.\frac{\partial W_{I I}\left(x_{I I}, t\right)}{\partial x_{I I}}\right|_{x_{I I}=0}=\left.\frac{\partial W_{1}\left(x_{1}, t\right)}{\partial x_{1}}\right|_{x_{1}=0}=0, \\
& U_{I}(0, t)=U_{I I}(0, t)=U_{1}(0, t)=0, \quad U_{I}(L, t)=U_{I I}(L, t)=U_{n}(l, t), \\
& \left.\frac{\partial W_{I}\left(x_{I}, t\right)}{\partial x_{I}}\right|_{x_{I}=L}=\left.\frac{\partial W_{I I}\left(x_{I I}, t\right)}{\partial x_{I I}}\right|_{x_{I I}=L}=\left.\frac{\partial W_{n}\left(x_{n}, t\right)}{\partial x_{n}}\right|_{x_{n}=l}, \\
& W_{n}(l, t)-\left.\left(R-l_{0}\right) \frac{\partial W_{n}\left(x_{n}, t\right)}{\partial x_{n}}\right|_{x_{n}=l}=0, \\
& \left.\sum_{k=I}^{I I}(E J)_{k} \frac{\partial^{3} W_{k}\left(x_{k}, t\right)}{\partial x_{k}^{3}}\right|_{x_{k}=L}+\left.(E J)_{n} \frac{\partial^{3} W_{n}\left(x_{n}, t\right)}{\partial x_{n}^{3}}\right|_{x_{n}=l}+ \\
& +\left(R-l_{0}\right)\left[\left.\sum_{k=I}^{I I}(E J)_{k} \frac{\partial^{2} W_{k}\left(x_{k}, t\right)}{\partial x_{k}{ }^{2}}\right|_{x_{k}=L}+\left.(E J)_{n} \frac{\partial^{2} W_{n}\left(x_{n}, t\right)}{\partial x_{n}{ }^{2}}\right|_{x_{n}=l}\right]-m \frac{\partial^{2} W_{n}(l, t)}{\partial t^{2}}=0, \\
& W_{j}(l, t)=W_{j+1}(0, t), \quad U_{j}(l, t)=U_{j+1}(0, t), \\
& \left.\frac{\partial W_{j}\left(x_{j}, t\right)}{\partial x_{j}}\right|_{x_{j}=l}=\left.\frac{\partial W_{j+1}\left(x_{j+1}, t\right)}{\partial x_{j+1}}\right|_{x_{j+1}=0}, \\
& \left.(E J)_{j} \frac{\partial^{2} W_{j}\left(x_{j}, t\right)}{\partial x_{j}^{2}}\right|_{x_{j}=l}=\left.(E J)_{j+1} \frac{\partial^{2} W_{j+1}\left(x_{j+1}, t\right)}{\partial x_{j+1}^{2}}\right|_{x_{j+1}=0}, \\
& \left.(E J)_{j} \frac{\partial^{3} W_{j}\left(x_{j}, t\right)}{\partial x_{j}^{3}}\right|_{x_{j}=l}=\left.(E J)_{j+1} \frac{\partial^{3} W_{j+1}\left(x_{j+1}, t\right)}{\partial x_{j+1}^{3}}\right|_{x_{j+1}=0}, \quad j=1,2, \ldots, n-1 .
\end{aligned}
$$

Due to the geometric nonlinearity of the analyzed system, the perturbation method (small parameter of the amplitude method) was used to solve the boundary problem. By substituting the solution of the differential equations of motion into the boundary conditions 
(8-18), the system of homogeneous equations is obtained which solution is a transcendental equation describing free vibrations of the column.

\section{Results of numerical computations}

Taking into consideration the mathematical model of the analyzed system, an original computational algorithms were developed. Results are presented in the dimensionless form after prior definition of the parameters of external $\lambda$ and bifurcation load $\lambda_{\mathrm{b}}$, frequency of vibrations $\Omega^{*}$ and the parameters describing the shape of the nonprismatic rod:

$$
\begin{gathered}
\lambda=\frac{P L^{2}}{(E J)_{p o r}}, \lambda_{b}=\frac{P_{b} L^{2}}{(E J)_{p o r}}, \Omega_{i}^{*}=\frac{(\rho A)_{p o r} \omega_{i}^{2} L^{4}}{(E J)_{p o r}}, \\
Z^{*}=\frac{b_{1}-b_{n}}{L}, p^{*}=\frac{p}{L}, q^{*}=\frac{q}{b_{p r}}, R^{*}=\frac{R-l_{0}}{L} .
\end{gathered}
$$
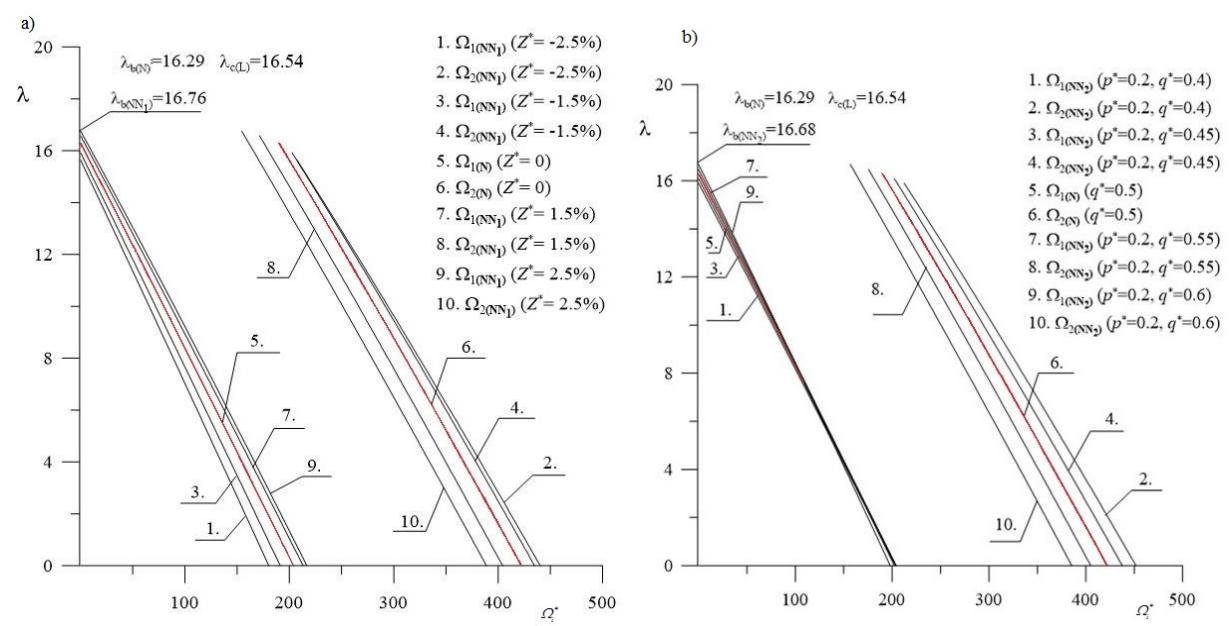

Fig. 2. The first two frequencies of vibrations as a function of external load parameter for selected values of the approximating parameters $\left(R^{*}=0.35, \mu=0.25\right)$.

A change of two first natural frequency parameters as a function of the external load for selected geometry of the loading structure was presented in figure 2 . The values of load for which the frequency reaches the zero value, refer to the bifurcation load of the system (the structure lost its rectilinear form of the static equilibrium). Taking into account the element of the variable cross-section in the physical model has got an influence on the value of the natural frequency parameter and it may be used for control of the dynamic properties of the column. Properly selected parameters of the approximating functions coefficients may also cause an increase in the bifurcation load compared with the reference system made of prismatic rods (compare fig. 3). 


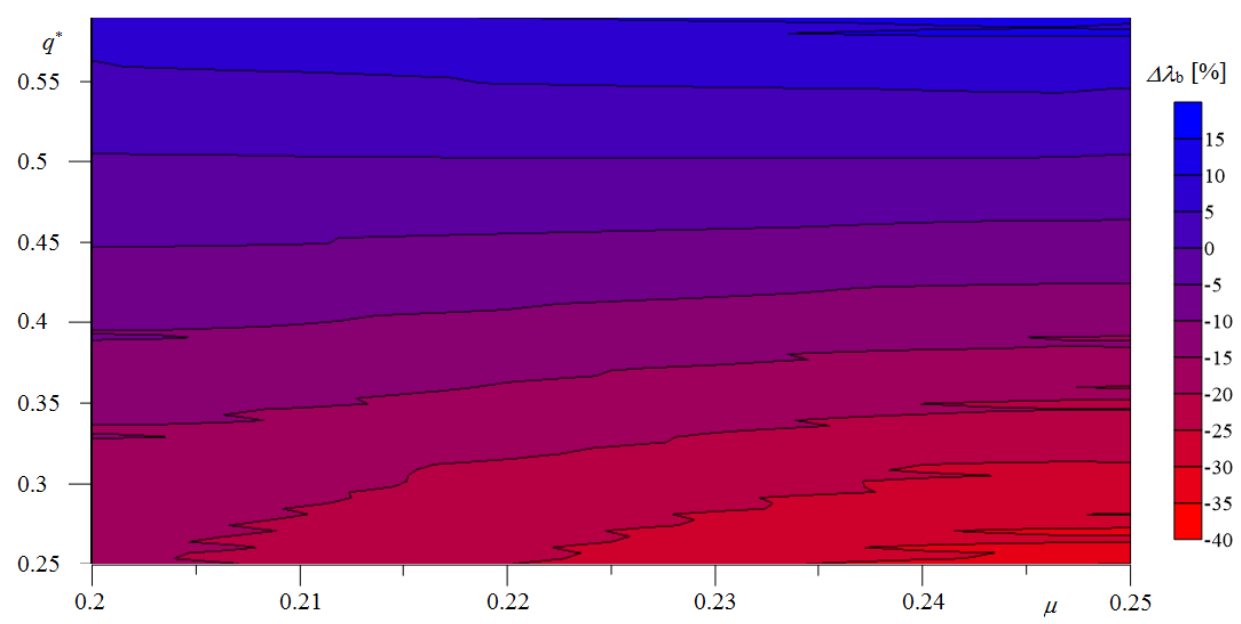

Fig. 3. An increase of the bifurcation load of the geometrically nonlinear column with nonprismatic rod in relation to the asymmetry of the flexural stiffness coefficient $\left(p^{*}=0.4, R^{*}=0.087\right)$.

\section{Conclusions}

After taking into account in the model of the geometrically nonlinear column the rod of variable cross-section, a change in the value of the natural frequency of vibrations and bifurcation load was noted in comparison with the systems built of prismatic elements. It was proved that the appropriately chosen values of the parameters describing the nonprismatic rod outline can be used for to control the dynamic properties and increase the strength of the system.

The calculation model proposed in this work and the algorithm developed on its basis is universal and enables a quick analyze od complex beam systems with nonprismatic elements described by any approximating functions, realizing different types of external loading.

\section{Acknowledgements}

The study has been carried out within the framework of project BS/MN 1-101-302/17/P realized at Częstochowa University of Technology.

\section{References}

1. S. Uzny, K. Sokół, M. Osadnik, Eng. Mech Conf. Proceedia $(562-565,2016)$

2. K. Sokół, A. Kulawik, J. Appl. Math. Comput. Mech., 13(1), 115-124 (2014)

3. W. Sochacki, J. of Vibroeng. 15(1), 280-290 (2013)

4. J.T. Katsikadelis, G.C. Tsiatas, Intern. J. of Non-lin. Mech., 42, 164-171 (2007)

5. J.T. Katsikadelis, G.C. Tsiatas, Intern. J. of Mech. Sc., 49, 1204-1212 (2007)

6. R. Bogacz, S. Imiełowski, L. Tomski, Dyn. Of Continua Intern (Symp., 1996)

7. L. Tomski, J. Szmidla, S. Uzny, Thin-Wall. Struct., 45, 945-949 (2007)

8. L. Tomski, S. Uzny, Intern. J. Sol. Struct., 45, 87-112 (2008) 